\title{
Intrahepatic cholestasis of pregnancy is associated with an increased risk of gestational diabetes and preeclampsia
}

\author{
Congcong Liu ${ }^{1}$, Jinsong Gao ${ }^{1}$, Juntao Liu ${ }^{1}$, Xietong Wang ${ }^{2}$, Jing $\mathrm{He}^{3}$, Jingxia Sun ${ }^{4}$, Xiaowei Liu ${ }^{5}$, \\ Shixiu Liao ${ }^{6}$
}

${ }^{1}$ Department of Obstetrics and Gynecology, Peking Union Medical College Hospital, Peking Union Medical College, Chinese Academy of Medical Sciences, Beijing, China; ${ }^{2}$ Department of Obstetrics and Gynecology, Shandong Provincial Hospital Affiliated to Shandong University, Jinan, China; ${ }^{3}$ Department of Obstetrics and Gynecology, Women's Hospital School of Medicine Zhejiang University, Hangzhou, China; ${ }^{4}$ Department of Obstetrics and Gynecology, the First Clinical Hospital affiliated to Harbin Medical University, Harbin, China; ${ }^{5}$ Department of Obstetrics and Gynecology, Beijing Obstetrics and Gynecology Hospital Capital Medical University, Beijing, China; ${ }^{6}$ Department of Obstetrics and Gynecology, Henan Provincial People's Hospital, Zhengzhou, China

Contributions: (I) Conception and design: J Gao, J Liu, C Liu; (II) Administrative support: None; (III) Provision of study materials or patients: J Liu, X Wang, J He, X Liu, S Liao; (IV) Collection and assembly of data: C Liu; (V) Data analysis and interpretation: J Gao, J Liu, C Liu; (VI) Manuscript writing: All authors; (VII) Final approval of manuscript: All authors.

Correspondence to: Jinsong Gao; Juntao Liu. Department of Obstetrics and Gynecology, Peking Union Medical College Hospital, Peking Union Medical College, Chinese Academy of Medical Sciences, No. 1 Shuai Fuyuan Road, Dongcheng District, Beijing 100730, China. Email: gaojingsong@pumch.cn; liujtpumch@sina.com.

Background: Previous studies have shown that intrahepatic cholestasis of pregnancy (ICP) is associated with an increased incidence of adverse perinatal outcomes, and this supports the contention that ICP is associated with increased risk for both gestational diabetes mellitus (GDM) and preeclampsia. The purpose of this study was to review adverse maternal and perinatal outcomes of ICP in the Chinese population, and to investigate the association between ICP and GDM, as well as between ICP and preeclampsia.

Methods: We conducted a retrospective cohort study in which we compared pregnancies affected by ICP with all other deliveries during the study period. Data from women with singleton pregnancies who delivered in 14 representative hospitals in China between October 1, 2016 and September 30, 2017 were collected from our database system. We then performed logistic regression analysis to determine the odds ratios (OR) and $95 \%$ CIs of the adverse pregnancy outcomes among women with or without ICP.

Results: A total of 95,728 singleton births were included in the study, and among these, 911 pregnancies were diagnosed as having ICP, resulting in an incidence of $0.95 \%$. Women with ICP were more likely to have GDM [adjusted odds ratio (aOR), 1.406; 95\% CI, 1.179-1.677; $\mathrm{P}<0.001$ ] and preeclampsia (aOR, 2.241; 95\% CI, 1.678-2.992; P<0.001) compared with those who did not have ICP. Women in the ICP group exhibited higher rates of scheduled cesarean deliveries (aOR, 3.527; 95\% CI, 2.981-4.173; $\mathrm{P}<0.001$ ) and cesarean deliveries during labor (aOR, 4.388; 95\% CI, 1.815-10.612; $\mathrm{P}=0.027$ ). Women with ICP were also more likely to have iatrogenic preterm delivery (aOR, 2.449; 95\% CI, 1.92-3.122; $\mathrm{P}<0.001$ ) and admission to the neonatal intensive care unit (aOR, 1.572; 95\% CI, 1.318-1.874; $\mathrm{P}<0.001$ ). There was no increased risk of stillbirth in the cohort of ICP cases (aOR, 0.430; 95\% CI, 0.049-3.767; P=0.259).

Conclusions: ICP was associated with an increased risk of GDM and preeclampsia in singleton pregnancies. Pregnancies with ICP therefore have significantly increased risks of adverse perinatal outcomes.

Keywords: Intrahepatic cholestasis of pregnancy (ICP); preeclampsia; gestational diabetes; bile acids; stillbirth

Submitted Jun 22, 2020. Accepted for publication Sep 25, 2020.

doi: $10.21037 /$ atm-20-4879

View this article at: http://dx.doi.org/10.21037/atm-20-4879 


\section{Introduction}

Intrahepatic cholestasis of pregnancy (ICP) is a pregnancy-specific liver disease (1) that is characterized by pruritus, elevated total bile acids (TBAs), and abnormal liver function tests-usually presenting in the second or third trimester. The pathogenesis of ICP remains poorly understood and may be associated with genetic, hormonal, immunologic and/or environmental factors (2-4). The prevalence of ICP seems to vary significantly among different ethnic and geographic populations, with a reported incidence ranging from $0.1 \%$ to $15.6 \%$ $(5,6)$. For example, the incidence of ICP is $1-4 \%$ in the Yangtze River region, while the incidence in Chongqing and Chengdu is greater than $5 \%$ (7). There is currently no international consensus regarding the diagnosis and treatment of ICP, and inconsistent data in the literature have led to varied practices in the management of ICP.

Previous investigators have concluded that ICP is associated with an increased risk for spontaneous and iatrogenic preterm labor, respiratory distress syndrome (RDS), meconium-stained amniotic fluid (MSAF), neonatal intensive care unit (NICU) admission, and stillbirth (8-10).

Both preeclampsia and gestational diabetes mellitus (GDM) exert an impact on the mother and the fetus. In 2013, Wikström et al. were the first to systematically describe highly significant associations between ICP and both GDM and preeclampsia (9). The association of ICP with GDM and preeclampsia was evaluated recently in several studies (9,11-16), although there are few studies on the association of ICP with GDM and preeclampsia. Moreover, there is a high degree of heterogeneity between ICP and GDM studies, and a relative lack of reliable data. In this study we aimed to review adverse maternal and perinatal outcomes of ICP in the Chinese population, and to investigate the association between ICP and the risk of gestational diabetes and preeclampsia.

We present the following article in accordance with the STROBE reporting checklist (available at http://dx.doi. org/10.21037/atm-20-4879).

\section{Methods}

We conducted a retrospective cohort study in which women diagnosed with ICP were compared with respect to all other deliveries during the study period. The incidences of GDM, preeclampsia, and adverse perinatal outcomes in each group were subsequently compared.

\section{Ethical statement}

Our study was performed in compliance with the Declaration of Helsinki (as revised in 2013). Our study method was approved by the Peking Union Medical College Hospital Review Board (reference number: JS-1151). The need for written informed consent was waived because of the retrospective nature of the study, and the dataset was deidentified in order to protect patient privacy.

\section{Patient selection and data collection}

This study was conducted at 14 representative hospitals in 10 provinces from 4 major economic zones in China (including 2 secondary and 12 tertiary hospitals, 7 general hospitals, and 7 women's and children's hospitals) between October 1, 2016 and September 30, 2017. Specially assigned individuals at each hospital were responsible for collecting and reporting information through an Internetbased system using a data collection system encompassing pregnant women who delivered. All deliveries in the hospitals after 20 weeks of gestation with a diagnosis of ICP were included in the study group, and ICP was diagnosed according to the 2015 guidelines of the Chinese Medical Association of Obstetrics and Gynecology (17). The diagnosis of ICP was based on pruritus without itching skin disease, elevated liver enzymes, and elevated levels of maternal serum TBA $(\geq 10 \mu \mathrm{mol} / \mathrm{L})$. All pregnancies that were complicated by severe congenital malformations such as chromosomal abnormalities and/or multiple congenital anomalies, all twin and higher multiple pregnancies, and cases with missing data were excluded from the study. The control groups consisted of all other pregnancies without ICP delivered at the aforementioned hospitals over the same time-period. We chose eligible cases to input into the data collection system and exported them from the central database system. In order to protect the privacy of patients, personal identifying information for all cases was removed during analysis. This study was approved by the Peking Union Medical College Hospital Review Board (reference number: JS-1151), which waived informed consent.

We collected data for each case and control, including maternal characteristics, maternal age, gravidity, parity, pregestational body mass index (BMI), assisted reproductive technology (ART), diagnosed GDM, and diagnosed preeclampsia; as well as gynecologic and obstetric history, major medical history, and major family history. The perinatal outcomes included gestational age at birth, 
Table 1 Maternal characteristics for singleton pregnancies with or without ICP

\begin{tabular}{lccc}
\hline Characteristic & ICP $(\mathrm{n}=911)$ & No ICP $(\mathrm{n}=94,817)$ & $\mathrm{P}$ values \\
\hline Maternal age, years & $30.8 \pm 4.33$ & $30.68 \pm 4.55$ & 0.445 \\
Pre-pregnancy BMI, $\mathrm{kg} / \mathrm{m}^{2}$ & $21.68 \pm 15.88$ & $20.97 \pm 7.53$ & 0.177 \\
Gravidity, $\mathrm{n}^{*}$ & $2.21 \pm 1.31$ & $2.18 \pm 1.26$ & 0.559 \\
Parity, $\mathrm{n}$ * & $1.34 \pm 0.53$ & $1.42 \pm 0.57$ & $<0.001$ \\
ART, $\mathrm{n}(\%)$ & $38(4.2)$ & $2,105(2.2)$ & $<0.001$ \\
Gestational diabetes mellitus, $\mathrm{n}(\%)$ & $156(17.1)$ & $11,785(12.4)$ & $<0.001$ \\
Preeclampsia, $\mathrm{n}(\%)$ & $50(5.5)$ & $2,317(2.4)$ & $<0.001$ \\
Chronic hypertension, $\mathrm{n}(\%)$ & $6(0.7)$ & $383(0.4)$ & 0.229 \\
\hline
\end{tabular}

*, data are presented as mean \pm SD; ART, assisted reproductive technology; BMI, body mass index; ICP, intrahepatic cholestasis of pregnancy.

birthweight, induction of labor, the method of delivery, postpartum hemorrhage, preterm labor (iatrogenic and spontaneous), small for gestational age (SGA), MSAF, NICU admission, Apgar score, stillbirth, and neonatal death.

Preeclampsia was defined according to the guidelines of the American College of Obstetricians and Gynecologists (18). GDM was defined as a $75 \mathrm{~g}$ oral glucose tolerance test (OGTT) showing a fasting glucose level higher than $5.1 \mathrm{mmol} / \mathrm{L}(92 \mathrm{mg} / \mathrm{dL}), 1 \mathrm{~h}$ post-OGTT higher than $10.0 \mathrm{mmol} / \mathrm{L}(180 \mathrm{mg} / \mathrm{dL})$, or a 2 h post-OGTT higher than $8.5 \mathrm{mmol} / \mathrm{L}(153 \mathrm{mg} / \mathrm{dL})$ at $24-28$ weeks of pregnancy (19). Preterm labor was defined as delivery after 24 weeks and before 37 completed weeks of gestation. SGA was defined as birth weight $<10^{\text {th }}$ percentile, and a low Apgar score was defined as a score of $<7$ at 5 minutes after birth. Stillbirth was used to describe fetal deaths at 20 weeks of gestation or later, and neonatal death was defined as the death of an infant from 0 to 28 days after birth. The grading criteria for BMI were based on the recommendations of the Working Group on Obesity in China: underweight, BMI $<18.5 \mathrm{~kg} / \mathrm{m}^{2}$; normal weight, BMI 18.5 to $23.9 \mathrm{~kg} / \mathrm{m}^{2}$; overweight, BMI $24.0-27.9 \mathrm{~kg} / \mathrm{m}^{2}$; and obesity, BMI $\geq 28.0 \mathrm{~kg} / \mathrm{m}^{2}$ (20). Postpartum hemorrhage (PPH) was defined as a blood loss of $\geq 500 \mathrm{~mL}$ within $24 \mathrm{~h}$ after vaginal delivery or $\geq 1,000 \mathrm{~mL}$ after cesarean delivery. Severe PPH was defined as a blood loss of $\geq 1,000 \mathrm{~mL}$ within $24 \mathrm{~h}$ after delivery (21).

The primary outcome of this study was the incidence of GDM and preeclampsia among women with ICP. Secondary outcomes were the correlations between ICP and stillbirth, gestational age at birth, birthweight, induction of labor, mode of delivery, presence of postpartum hemorrhage, preterm labor (iatrogenic and spontaneous), SGA, MSAF, 5-min Apgar score, NICU admission, and neonatal death.

\section{Statistical analysis}

We performed all of the statistical analyses using SPSS (version 25.0, Chicago, IL, USA). Statistical significance was accepted at a $\mathrm{P}$ value $<0.05$. Continuous variables are presented as means \pm standard deviations, and differences between groups were assessed using a Student's $t$-test. Categorical variables are presented as counts and percentages, which were compared using Chi-squared or Fisher's exact-probability tests. We compared baseline characteristics and maternal and neonatal outcomes for women with and without ICP. Logistic regression was used to determine the odds ratios (OR) and $95 \% \mathrm{CI}$ of the adverse pregnancy outcomes among women with or without ICP, with the following confounders considered: maternal age, body mass index, parity, and chronic hypertension.

\section{Results}

A total of 95,728 singleton births were included in the present study; of these, 911 pregnancies were diagnosed as ICP, resulting in an incidence of $0.95 \%$ from October 1 , 2016 to September 30, 2017.

In our population, more than $99 \%$ of the women were of Han ethnicity, and only 2 pregnant women smoked. The primary maternal characteristics of the women with and without ICP are presented in Table 1. Maternal age and prepregnancy BMI did not differ between the groups. Women 
Table 2 Maternal characteristics of preeclampsia at presentation with ICP

\begin{tabular}{lccc}
\hline Characteristic & Preeclampsia $(\mathrm{n}=50)$ & No preeclampsia $(\mathrm{n}=861)$ & P values \\
\hline Maternal age, years & $30.74 \pm 4.40$ & $30.80 \pm 4.33$ & 0.920 \\
Pre-pregnancy BMI ${ }^{*}, \mathrm{n}(\%)$ & & & \\
Underweight/normal weight & $31(62.0)$ & $733(85.1)$ & $<0.001$ \\
Overweight & $17(34.0)$ & $110(12.8)$ & $<0.001$ \\
Obese & $2(4.0)$ & $18(2.1)$ & 0.965 \\
Nulliparous, $\mathrm{n}(\%)$ & $35(70.0)$ & $538(62.5)$ & 0.285 \\
ART, n (\%) & $7(14.0)$ & $31(3.6)$ & $<0.001$ \\
\hline
\end{tabular}

*, underweight, BMI $<18.5 \mathrm{~kg} / \mathrm{m}^{2}$; normal weight, BMI 18.5 to $23.9 \mathrm{~kg} / \mathrm{m}^{2}$; overweight, BMI 24.0 to $27.9 \mathrm{~kg} / \mathrm{m}^{2}$; and obesity, BMI $\geq 28.0 \mathrm{~kg} / \mathrm{m}^{2}$. ART, assisted reproductive technology; BMI, body mass index; ICP, intrahepatic cholestasis of pregnancy.

Table 3 Maternal characteristics of GDM at presentation with ICP

\begin{tabular}{|c|c|c|c|}
\hline Characteristic & GDM $(n=156)$ & No GDM (n=755) & $P$ values \\
\hline \multicolumn{4}{|l|}{ Pre-pregnancy BMI*, n (\%) } \\
\hline Underweight/normal weight & $118(75.6)$ & $646(85.6)$ & 0.002 \\
\hline Overweight & $32(20.5)$ & $95(12.6)$ & 0.009 \\
\hline Nulliparous, n (\%) & 91 (58.3) & $482(63.8)$ & 0.195 \\
\hline ART, n (\%) & $10(6.4)$ & $28(3.7)$ & 0.124 \\
\hline
\end{tabular}

*, Underweight, BMI <18.5 kg/m²; normal weight, BMI 18.5-23.9 kg/m²; overweight, BMl 24.0-27.9 kg/m²; and obesity, BMI $\geq 28.0 \mathrm{~kg} / \mathrm{m}^{2}$. ART, assisted reproductive technology; BMI, body mass index; GDM, gestational diabetes mellitus; ICP, intrahepatic cholestasis of pregnancy.

with ICP exhibited a higher proportion of conceptions with assisted reproduction ( $4.2 \%$ vs. $2.2 \%, \mathrm{P}<0.001)$.

The prevalence of GDM was significantly greater in the ICP group (17.1\% vs. 12.4\%; aOR, 1.406; 95\% CI, 1.179 1.677; $\mathrm{P}<0.001)$. Compared with the group without ICP, the incidence of preeclampsia was also significantly increased in the ICP group (5.5\% vs. 2.4\%; aOR, 2.241; 95\% CI, $1.678-$ 2.992; $\mathrm{P}<0.001)$. There were no significant differences in maternal age or nulliparous status between women with ICP who experienced preeclampsia and those who did not. Women with ICP who experienced preeclampsia had a significant increase in overweight cases $34.0 \%$ vs. $12.8 \%$, $\mathrm{P}<0.001)$, and a higher proportion conceived with assisted reproduction $(14 \%$ vs. $3.6 \%, \mathrm{P}<0.001)$ (Table 2). There were no significant differences in nulliparous status or ART between ICP patients who experienced GDM and those who did not. Women with ICP who experienced GDM were older $(32.08 \pm 4.56$ vs. $30.54 \pm 4.24, \mathrm{P}<0.001)$, and more likely to be overweight ( $20.5 \%$ vs. $12.6 \%, \mathrm{P}=0.009$; Table 3).

Maternal and neonatal outcomes of pregnancies with ICP are presented in Table 4. Women in the ICP group delivered earlier $(37.32 \pm 2.15$ vs. $38.49 \pm 2.01, \mathrm{P}<0.001)$, had lower birth weights $(3016.17 \pm 588.94$ vs. $3260.58 \pm 551.02$, $\mathrm{P}<0.001)$, and higher rates of SGA $(3.5 \%$ vs. $2.0 \%, \mathrm{P}=0.001)$ in our univariate analysis. However, after controlling for the confounding factors, these differences were no longer statistically significant.

The rates of scheduled cesarean deliveries $(74.3 \% v s$. 43.7\%; aOR, 3.527; 95\% CI, 2.981-4.173; $\mathrm{P}<0.001)$ and cesarean deliveries during labor (0.7\% vs. $0.3 \%$; aOR, 4.388; 95\% CI, 1.815-10.612; $\mathrm{P}=0.027)$ were significantly higher in the ICP group; as were the rates of iatrogenic preterm delivery (23.8\% vs. 5.6\%; aOR, 2.449; 95\% CI, 1.92-3.122; $\mathrm{P}<0.001)$. The rates of admission to the NICU $(29.5 \%$ vs. 
Table 4 Maternal and neonatal outcomes of pregnancies with ICP

\begin{tabular}{|c|c|c|c|c|}
\hline Characteristic & ICP $(n=911)$ & No ICP $(n=94,817)$ & $P$ value & aOR $(95 \% \mathrm{Cl})$ \\
\hline Birthweight, g & $3,016.17 \pm 588.94$ & $3,260.58 \pm 551.02$ & $<0.001$ & $1(1.000-1.000)$ \\
\hline Induction of labor, $\mathrm{n}(\%)$ & $37(4.1)$ & $6,355(6.7)$ & 0.001 & $1.292(0.884-1.889)$ \\
\hline \multicolumn{5}{|l|}{ Mode of delivery, $\mathrm{n}(\%)$} \\
\hline Scheduled cesarean delivery & $677(74.3)$ & $41,475(43.7)$ & $<0.001$ & $3.527(2.981-4.173)$ \\
\hline Cesarean delivery during labor & $6(0.7)$ & $258(0.3)$ & 0.027 & $4.388(1.815-10.612)$ \\
\hline Postpartum hemorrhage, n (\%) & $30(3.3)$ & $5,102(5.4)$ & 0.005 & $0.496(0.324-0.76)$ \\
\hline Severe postpartum hemorrhage, $n$ (\%) & $8(0.9)$ & $712(0.8)$ & 0.658 & $1.044(0.459-2.373)$ \\
\hline latrogenic preterm delivery, $\mathrm{n}(\%)$ & $217(23.8)$ & $5,296(5.6)$ & $<0.001$ & 2.449 (1.92-3.122) \\
\hline Spontaneous preterm delivery, $\mathrm{n}(\%)$ & $8(0.9)$ & $1,068(1.1)$ & 0.479 & $0.914(0.439-1.904)$ \\
\hline Small for gestational age, $\mathrm{n}(\%)$ & $32(3.5)$ & $1,879(2.0)$ & 0.001 & $0.569(0.385-0.843)$ \\
\hline Meconium-stained amniotic fluid, n (\%) & $35(3.8)$ & $2,990(3.2)$ & 0.237 & $1.246(0.883-1.76)$ \\
\hline 5-min Apgar score <7, n (\%) & $5(0.7)$ & $603(0.6)$ & 0.932 & $0.547(0.221-1.356)$ \\
\hline Stillbirth, n (\%) & $1(0.1)$ & $305(0.3)$ & 0.259 & $0.430(0.049-3.767)$ \\
\hline Neonatal death, n (\%) & $0(0)$ & $81(0.1)$ & 0.377 & 0 \\
\hline NICU admission, $\mathrm{n}(\%)$ & $269(29.5)$ & $12,835(13.5)$ & $<0.001$ & $1.572(1.318-1.874)$ \\
\hline
\end{tabular}

aOR, adjusted odds ratio; ICP, intrahepatic cholestasis of pregnancy; NICU, neonatal intensive care unit.

13.5\%; aOR, 1.572; 95\% CI, 1.318-1.874; P<0.001) were significantly elevated in the ICP group; $\mathrm{PPH}$ was, however, less frequent in the ICP group (3.3\% vs. 5.4\%; aOR, 0.496; 95\% CI, 0.324-0.76; P=0.005). There were no significant differences in severe PPH between women with ICP and those without $(0.9 \%$ vs. $0.8 \%$; aOR, 1.044 ; $95 \%$ CI, $0.459-$ 2.373; $\mathrm{P}=0.658)$. We observed no significant differences in the rates of spontaneous preterm delivery, MSAF, 5-min Apgar score $<7$, stillbirth, or neonatal death.

\section{Discussion}

\section{Main findings}

This retrospective study bears two main findings: i.e., that ICP demonstrates a strong association with GDM and preeclampsia (the prevalence of ICP in our population was $0.95 \%$ ), and that women with ICP have significantly increased risks for adverse perinatal outcomes.

\section{Interpretation}

In this study we showed a higher risk of GDM and preeclampsia among women with ICP compared with those without, which is consistent with previous studies $(9,15)$. Marathe et al. (15) found that ICP was associated with a higher incidence of GDM (OR 3.06) and preeclampsia (OR 75.84), and Martineau et al. (12) reported that the incidence of GDM was higher following the onset of ICP (OR 4.69). Raz et al. (14) found a higher incidence of preeclampsia in 54 singleton pregnancies with ICP compared with pregnancies without ICP, and preeclampsia usually occurred 2-4 weeks after the diagnosis of ICP.

The mechanism(s) underlying the direct relation of ICP to GDM and preeclampsia remains unclear. Martineau et al. (16) reported that ICP is characterized by glucose intolerance and dyslipidemia, consistent with metabolic syndrome. Women with ICP exhibited higher triglycerides, total cholesterol, and low-density lipoprotein cholesterol; 
and lower high-density lipoprotein cholesterol—and these indices were also associated with an increased risk for preeclampsia and GDM $(22,23)$. Women with a higher BMI and whose metabolic pathways are disrupted may also have an increased risk for adverse outcomes of pregnancy $(24,25)$. Our study also showed that the incidence of GDM and preeclampsia in overweight pregnant women with ICP was significantly increased.

Bile acid as a ligand-combined with farnesoid $\mathrm{X}$ receptor (FXR) and Takeda G protein-coupled receptor 5 (TGR5)—can broadly influence glucolipid metabolism $(26,27)$. Activated FXR inhibits gluconeogenesis, augments insulin secretion, and enhances insulin sensitivity (28-30). Activated TGR5 activates and induces the secretion of glucagon-like peptide 1 (GLP-1) by intestinal endocrine cells, and GLP-1 can then promote insulin secretion (31). Bile acid can also directly stimulate TGR5 in islet cells to promote insulin release (32). Of note, the increase in sulfated progesterone metabolites in women with ICP may antagonize FXR (33). The significant increase of GDM among women with ICP may thus be due to the decrease in FXR and TGR5 activities, which could lead to aberrant bile acid homeostasis.

High bile acid levels have also been shown to cause vasoconstriction of the chorionic plate veins which leads to structural damage of the placenta, causing reduced oxygen (34). High bile acid levels can also cause impairment of the placental antioxidant system and oxidative damage, inducing an increase in the number of placental syncytial knots $(35,36)$; and increased numbers of syncytial knots have been reported in the placenta of pregnancies complicated by preeclampsia (36). Bile acids also contribute to endothelial damage in the kidneys and lungs through the production and release of reactive oxygen species, which can lead to oxidative stress. Oxidative stress can then promote the formation of a variety of vasoactive mediators, including endoglin-1, soluble fms-like tyrosine kinase receptor (sFlt-1), and others (37). Previous studies showed that endoglin and sFlt-1 were also increased in preeclampsia $(38,39)$.

We observed a significant correlation between ICP and adverse perinatal outcomes, and investigators previously reported that ICP was associated with an increased risk of stillbirth $(8,40)$. ICP is characterized by fetal death without any overt cause, but because there is currently no specific way to monitor the fetus, it is therefore often terminated in advance to avoid adverse pregnancy outcomes. With effective management during pregnancy, there was no increase in the incidence of stillbirth in our study-consistent with previously published studies $(9,15)$. However, the rates of cesarean section, iatrogenic premature delivery, and NICU admission increased significantly. Although optimal delivery timing with respect to ICP is challenging given the paucity of high-quality studies, the Royal College of Obstetrics and Gynecology does not recommend active management (41). Recently, a meta-analysis showed that the risk of stillbirth was increased in women with ICP when serum bile acid concentrations were $100 \mu \mathrm{mol} / \mathrm{L}$ or more (42), but bile acid was not always a reliable monitoring index (43). The current study was not able to evaluate the levels of serum bile acids. We recommend that clinicians bolster their monitoring of bile acid levels, and make an individualized judgment as to whether the risks of preterm labor outweigh the risks of disease.

Our study had a lower frequency of PPH compared with other studies. Marathe et al. defined PPH as a blood loss of more than $500 \mathrm{~mL}$ in the $24 \mathrm{~h}$ after delivery, and the incidence of $\mathrm{PPH}$ was $30 \%$ in ICP patients. In our study, pregnancies had higher rates of cesarean deliveries in the ICP group, and we defined PPH as a blood loss of $\geq 1,000 \mathrm{~mL}$ after cesarean delivery within $24 \mathrm{~h}$. Our study's lower frequency of PPH might be explained by the different definitions used or by blood loss underestimations.

\section{Strengths and limitations}

This study was a multicenter, retrospective cohort study with large samples of ICP patients, which indicates the prevalence and characteristics of ICP in the Chinese population. There were also some limitations. This was a retrospective study, which may raise some concerns regarding data quality; and regarding the risks of selection and information bias. We were not able to retrieve the specific indicators needed to assess disease severity or onset time, such as serum bile acid or transaminase levels. We were also unable to establish a relationship for onset time between ICP and GDM or ICP and preeclampsia, and could not evaluate a dose-dependent relationship between bile acid concentrations and the risk for GDM and preeclampsia.

\section{Conclusions}

This was a multicenter cohort study concerning women with ICP in China. Although a strong association was 
found between ICP and GDM as well as between ICP and preeclampsia, ICP did not increase the risk of stillbirth. It is thus necessary to further analyze the timeframe for any relationships between ICP and GDM or preeclampsia, and to better understand the potential mechanism(s) of any correlations. We need to strengthen the management and surveillance of women with ICP and control the weight of pregnant women in order to detect and treat preeclampsia as early as possible-thereby improving fetal outcomes and maternal prognosis.

\section{Acknowledgments}

We would like to thank the following for their support of this study: Hunan Maternal and Child Health Care Hospital; Nanfang Hospital Southern Medical University; Women's Hospital School of Medicine, Zhejiang University; Henan Provincial People's Hospital; Harbin Nangang District Maternity Hospital; Beijing Obstetrics and Gynecology Hospital, Capital Medical University; Shandong Provincial Hospital Affiliated to Shandong University; Beijing Haidian Maternal and Child Health Hospital; Sichuan University West China Second Hospital; Northwest Women and Children's Hospital; Ruijin Maternal and Child Health Hospital; Shengjing Hospital affiliated to China Medical University; and The First Clinical Hospital affiliated to Harbin Medical University. We thank LetPub (www.letpub.com) for its linguistic assistance during the preparation of this manuscript.

Funding: This work was supported by the $13^{\text {th }}$ Five-Year National Science and Technology Support Program (No. 2015BAI13B04) and the Chinese Academy of Medical Sciences (CAMS) Innovation Fund for Medical Science (CIFMS, No. 2017-I2M-3-007).

\section{Footnote}

Reporting Checklist: The authors have completed the STROBE reporting checklist. Available at http://dx.doi. org/10.21037/atm-20-4879

Data Sharing Statement: Available at http://dx.doi. org/10.21037/atm-20-4879

Conflicts of Interest: All authors have completed the ICMJE uniform disclosure form (available at http://dx.doi. org/10.21037/atm-20-4879). The authors have no conflicts of interest to declare.
Ethical Statement: The authors are accountable for all aspects of the work in ensuring that questions related to the accuracy or integrity of any part of the work are appropriately investigated and resolved. Our study was performed in compliance with the Declaration of Helsinki (as revised in 2013). This study method was approved by the Peking Union Medical College Hospital Review Board (No. JS-1151). The need for written informed consent was waived because of the retrospective nature of the study, and the data set was deidentified in order to protect patient privacy.

Open Access Statement: This is an Open Access article distributed in accordance with the Creative Commons Attribution-NonCommercial-NoDerivs 4.0 International License (CC BY-NC-ND 4.0), which permits the noncommercial replication and distribution of the article with the strict proviso that no changes or edits are made and the original work is properly cited (including links to both the formal publication through the relevant DOI and the license). See: https://creativecommons.org/licenses/by-nc-nd/4.0/.

\section{References}

1. Joshi D, James A, Quaglia A, et al. Liver disease in pregnancy. Lancet 2010;375:594-605.

2. Larson SP, Kovilam O, Agrawal DK. Immunological basis in the pathogenesis of intrahepatic cholestasis of pregnancy. Expert Rev Clin Immunol 2016;12:39-48.

3. Geenes V, Williamson C. Intrahepatic cholestasis of pregnancy. World J Gastroenterol 2009;15:2049-66.

4. Lammert F, Marschall HU, Glantz A, et al. Intrahepatic cholestasis of pregnancy: molecular pathogenesis, diagnosis and management. J Hepatol 2000;33:1012-21.

5. Laifer SA, Stiller RJ, Siddiqui DS, et al. Ursodeoxycholic acid for the treatment of intrahepatic cholestasis of pregnancy. J Matern Fetal Med 2001;10:131-5.

6. Lee RH, Goodwin TM, Greenspoon J, et al. The prevalence of intrahepatic cholestasis of pregnancy in a primarily Latina Los Angeles population. J Perinatol 2006;26:527-32.

7. Li L, Zhao XY, Ou XJ, et al. Clinical analysis of intrahepatic cholestasis of pregnancy. Zhonghua Gan Zang Bing Za Zhi 2013;21:295-8.

8. Geenes V, Chappell LC, Seed PT, et al. Association of severe intrahepatic cholestasis of pregnancy with adverse pregnancy outcomes: a prospective population-based casecontrol study. Hepatology 2014;59:1482-91. 
9. Wikström Shemer E, Marschall HU, Ludvigsson JF, et al. Intrahepatic cholestasis of pregnancy and associated adverse pregnancy and fetal outcomes: a 12-year population-based cohort study. BJOG 2013;120:717-23.

10. Arthuis C, Diguisto C, Lorphelin H, et al. Perinatal outcomes of intrahepatic cholestasis during pregnancy: an 8-year case-control study. PLoS One 2020;15:e0228213.

11. Arafa A, Dong JY. Association between intrahepatic cholestasis of pregnancy and risk of gestational diabetes and preeclampsia: a systematic review and meta-analysis. Hypertens Pregnancy 2020;39:354-60.

12. Martineau M, Raker C, Powrie R, et al. Intrahepatic cholestasis of pregnancy is associated with an increased risk of gestational diabetes. Eur J Obstet Gynecol Reprod Biol 2014;176:80-5.

13. Mor M, Shmueli A, Krispin E, et al. Intrahepatic cholestasis of pregnancy as a risk factor for preeclampsia. Arch Gynecol Obstet 2020;301:655-64.

14. Raz Y, Lavie A, Vered Y, et al. Severe intrahepatic cholestasis of pregnancy is a risk factor for preeclampsia in singleton and twin pregnancies. Am J Obstet Gynecol 2015;213:395.e1-8.

15. Marathe JA, Lim WH, Metz MP, et al. A retrospective cohort review of intrahepatic cholestasis of pregnancy in a South Australian population. Eur J Obstet Gynecol Reprod Biol 2017;218:33-8.

16. Martineau MG, Raker C, Dixon PH, et al. The metabolic profile of intrahepatic cholestasis of pregnancy is associated with impaired glucose tolerance, dyslipidemia, and increased fetal growth. Diabetes Care 2015;38:243-8.

17. Obstetrics Subgroup, Chinese Society of Obstetrics and Gynecology, Chinese Medical Association. Guidelines for diagnosis and treatment of intrahepatic cholestasis of pregnancy (2015). Zhonghua Fu Chan Ke Za Zhi 2015;50:481-5.

18. American College of Obstetricians and Gynecologists. Hypertension in pregnancy. Report of the American College of Obstetricians and Gynecologists' task force on hypertension in pregnancy. Obstet Gynecol 2013;122:1122-31.

19. American Diabetes Association. Standards of medical care in diabetes. Diabetes Care 2013;36:S11-66.

20. Zhou BF. Predictive values of body mass index and waist circumference for risk factors of certain related diseases in Chinese adults--study on optimal cut-off points of body mass index and waist circumference in Chinese adults. Biomed Environ Sci 2002;15:83-96.

21. Obstetrics Subgroup, Chinese Society of Obstetrics and
Gynecology, Chinese Medical Association. Guideline of prevention and treatment about postpartum hemorrhage (2014). Zhonghua Fu Chan Ke Za Zhi 2014;49:641-6.

22. Demirci O, Tugrul AS, Dolgun N, et al. Serum lipids level assessed in early pregnancy and risk of pre-eclampsia. J Obstet Gynaecol Res 2011;37:1427-32.

23. Jin WY, Lin SL, Hou RL, et al. Associations between maternal lipid profile and pregnancy complications and perinatal outcomes: a population-based study from China. BMC Pregnancy Childbirth 2016;16:60.

24. Somprasit C, Tanprasertkul C, Rattanasiri T, et al. High pre-pregnancy body mass index and the risk of poor obstetrics outcomes among Asian women using BMI criteria for Asians by World Health Organization Western Pacific Region (WPRO): a large cohort study. J Med Assoc Thai 2015;98:S101-7.

25. Cnattingius S, Bergstrom R, Lipworth L, et al. Prepregnancy weight and the risk of adverse pregnancy outcomes. N Engl J Med 1998;338:147-52.

26. Trauner M, Claudel T, Fickert P, et al. Bile acids as regulators of hepatic lipid and glucose metabolism. Dig Dis 2010;28:220-4.

27. Kim H, Fang S. Crosstalk between FXR and TGR5 controls glucagon-like peptide 1 secretion to maintain glycemic homeostasis. Lab Anim Res 2018;34:140-6.

28. Fang S, Suh JM, Reilly SM, et al. Intestinal FXR agonism promotes adipose tissue browning and reduces obesity and insulin resistance. Nat Med 2015;21:159-65.

29. Trabelsi MS, Daoudi M, Prawitt J, et al. Farnesoid X receptor inhibits glucagon-like peptide- 1 production by enteroendocrine L cells. Nat Commun 2015;6:7629.

30. Hou Y, Fan W, Yang W, et al. Farnesoid X receptor: an important factor in blood glucose regulation. Clin Chim Acta 2019;495:29-34.

31. Brighton CA, Rievaj J, Kuhre RE, et al. Bile Acids Trigger GLP-1 release predominantly by accessing basolaterally located $\mathrm{G}$ protein-coupled bile acid receptors. Endocrinology 2015;156:3961-70.

32. Kumar DP, Rajagopal S, Mahavadi S, et al. Activation of transmembrane bile acid receptor TGR5 stimulates insulin secretion in pancreatic $\beta$ cells. Biochem Biophys Res Commun 2012;427:600-5.

33. Abu-Hayyeh S, Papacleovoulou G, Lovgren-Sandblom A, et al. Intrahepatic cholestasis of pregnancy levels of sulfated progesterone metabolites inhibit farnesoid $\mathrm{X}$ receptor resulting in a cholestatic phenotype. Hepatology 2013;57:716-26.

34. Sepúlveda WH, González C, Cruz MA, et al. 
Vasoconstrictive effect of bile acids on isolated human placental chorionic veins. Eur J Obstet Gynecol Reprod Biol 1991;42:211-5.

35. Marín JJ, Macías RI, Briz O, et al. Molecular bases of the excretion of fetal bile acids and pigments through the fetal liver-placenta-maternal liver pathway. Ann Hepatol 2005;4:70-6.

36. Heazell AEP, Moll SJ, Jones CJ, et al. Formation of syncytial knots is increased by hyperoxia, hypoxia and reactive oxygen species. Placenta 2007;28:S33-40.

37. Perez MJ, Briz O. Bile-acid-induced cell injury and protection. World J Gastroenterol 2009;15:1677-89.

38. Leaños-Miranda A, Navarro-Romero CS, Sillas-Pardo LJ, et al. Soluble Endoglin As a Marker for Preeclampsia, Its Severity, and the Occurrence of Adverse Outcomes. Hypertension 2019;74:991-7.

39. Levine RJ, Maynard SE, Qian C, et al. Circulating angiogenic factors and the risk of preeclampsia. $\mathrm{N}$ Engl J

Cite this article as: Liu C, Gao J, Liu J, Wang X, He J, Sun J, Liu X, Liao S. Intrahepatic cholestasis of pregnancy is associated with an increased risk of gestational diabetes and preeclampsia. Ann Transl Med 2020;8(23):1574. doi: 10.21037/ atm-20-4879
Med 2004;350:672-83.

40. Williamson C, Hems LM, Goulis DG, et al. Clinical outcome in a series of cases of obstetric cholestasis identified via a patient support group. BJOG 2004;111:676-81.

41. Bicocca MJ, Sperling JD, Chauhan SP. Intrahepatic cholestasis of pregnancy: review of six national and regional guidelines. Eur J Obstet Gynecol Reprod Biol 2018;231:180-7.

42. Ovadia C, Seed PT, Sklavounos A, et al. Association of adverse perinatal outcomes of intrahepatic cholestasis of pregnancy with biochemical markers: results of aggregate and individual patient data meta-analyses. Lancet 2019;393:899-909.

43. Sentilhes L, Verspyck E, Pia P, et al. Fetal death in a patient with intrahepatic cholestasis of pregnancy. Obstet Gynecol 2006;107:458-60. 